\title{
A Comparison of the Immediate Effect of Swedish Massage vs Passive Stretching on Range of Motion in Overhead Athletes
}

\author{
Jim Schilling', Sujeiry Guzman', Chad Lewis', Jupil Ko ${ }^{2 *}$ \\ ${ }^{1}$ Northern Arizona University, Phoenix AZ, U.S \\ ${ }^{2}$ Incheon National University, Incheon, Republic of Korea
}

Received: April 28, 2020

Accepted: June 26, 2020

Published online: July 31,2020

Keywords:

Glenohumeral Joint

Golgi Tendon

Manual Therapy

Muscle Spindle

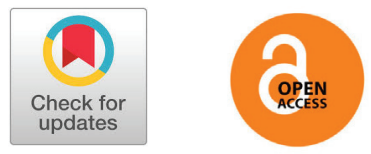

\section{ABSTRACT}

OBJECTIVES To compare the clinical outcome and effectiveness of Swedish massage and a traditional passive stretching program, on the shoulder of ROM in overhead athletes.

PARTICIPANTS A total of 10 overhead throwing overhead male athletes (age $=23.5 \pm 1.5 \mathrm{yrs}$; height $=186.69$ $\pm 6.35 \mathrm{~cm}$; mass $=91.86 \pm 12.48 \mathrm{~kg}$ ).

METHODS Participants were randomly assigned to either Swedish massage or a traditional passive static stretching program for 1 session of 5 minutes. Baseline and post-intervention shoulder ROM to end range in the flexed, external rotation, internal rotation, horizontal adduction and abduction positions. The Pearson chi-square test was applied to compare the differences of shoulder ROM of both therapeutic variables. Recorded outcome measures on the participants' shoulder ROM (flexion with scapula fixed, horizontal adduction, horizontal abduction, internal rotational \& external rotation) pre- \& post-interventions.

RESULTS Results indicate that participants in the Swedish massage therapeutic intervention group had significant improvements in their internal rotation post-treatment $(M=5.20, S D=1.304, p=.038)$ and the traditional passive stretching protocol group had significant improvements in shoulder external rotation post-treatment $(M=11.20, S D=2.68, p=.016)$.

CONCLUSION Swedish massage had better effects in four out of the five shoulder motions measured compared to passive static stretching. Educating overhead athletes on the potential beneficial effects of massage therapy and its role in maintaining shoulder range of motion.

( T) The Asian Society of Kinesiology and the Korean Academy of Kinesiology

\section{Introduction}

An epidemiological survey of 372 overhead athletes between the ages of 25 to 40 years of age no need to include the journal or authors, just cite indicated 43.8 percent of the athletes surveyed suffered from shoulder pain or injuries which involved intervention [1]. Overhead athletes are often participating in great overhead movements without

*Correspondence: Jupil Ko PhD, ATC, 119 Academy-ro, Yeonsu-gu, Incheon, Republic of Korea 22012; Tel: +82-32-835-8587; Fax: +82-32-835-0789; Email: jupilko@gmail.com the guidance of proper mechanics and conditioning directly contributing to decreases in shoulder range of motion [1]. Previous literature has established correlated increases in acute and chronic shoulder injury rates to individuals who suffer from a loss in shoulder range of motion [2]. Research involving Swedish massage techniques has solely been focused around general populations of people and has shown to provide positive (sounds like wording of the author) effects on delayed onset muscle soreness while also contributing to increases in joint range of motion through its ability to stimulate blood 
flow to muscle tissue [3]. Static stretching routines have been used as a means of increasing muscle laxity, flexibility, and joint range of motion among college and professional athletic populations as a means to prevent and rehabilitate an injury [4]. Research on overhead athletes and which interventions can facilitate immediate beneficial results shoulder range of motion are limited in number; this study aims to fill the gap in the literature. The purpose of this study is to determine if there is a significant difference in the range of motion in overhead athletes (softball and baseball players) after Swedish massage and a traditional passive stretching program.

Massage therapy is a common form of intervention to improve shoulder function through its therapeutical effects of alleviating pain, making it a frequent therapy among clinical populations as a method to improve shoulder range of motion $[3,5]$. Massage is a therapeutical intervention with variations in techniques or methods all separately contributing to a wide range of benefits [5]. Previous literature on manual therapies such as Swedish massage has lacked a defined description of their effectiveness in the areas of prevention and rehabilitation [5]. Swedish massage targets an increase in proprioception via the muscle fibers during the long strokes of the effleurage technique. The long strokes contribute to de-sensitizing muscle spindle fiber and increasing the sensitivity of the muscles Golgi-tendon organ (GTO) [6,7]. There is evidence that massage therapy promotes the excitation of neuromuscular interactions and inhibits muscle pain [6]. Although there have been studies done on the de-sensitizing of muscle spindles contributing to an increase in GTO activation as it correlates to pain, there is little research on how it affects range of motion [7].

Static stretching techniques are widely used among the overhead sports populations as a tool to increase shoulder range of motions through a decrease in muscle tension. A study conducted in 2017, compared static stretching and dynamic stretching techniques have been recently critiqued in views to which has a better effect on sports performance through an optimal length-tension relationship [8]. The study found, the use of static stretching led to a higher range of motion but did not lead to an optimal anaerobic effect with sports specific exercise in comparison to dynamic stretching protocols [8]. A second study found dynamic stretching to be most beneficial in increasing sarcomere length to optimally allow from joint range of motion contributing to an increased muscle spindle and GTO activation through a rebound principle [9]. Static stretching, although useful for overall muscle range of motion, has been proven to inhibit the athletes from their optimal performance and decrease muscle spindle and GTO innervation which could directly contribute to an increase in athletic injury [9]. We hypothesize that Swedish massage will have an equal to or more significant effect in the range of motion in overhead athletes than a traditional passive stretching program.

\section{Methods}

\section{Design Overview}

This study was a randomized controlled trial of Swedish massage and passive static stretching groups. The study was administered in a laboratory at Northern Arizona University (NAU) Biomedical campus and was securely approved to be conducted by the Institutional Review Board (IRB at NAU). Ten overhead athletes were divided into two groups using a computer-based application utilizing the block randomization method. Five participants received Swedish massage, and five participants received a traditional passive static stretching program. The study was conducted within one day, taking approximately 30 minutes to complete. The study was conducted by two primary investigators who were not blinded to group assignment. ROM measurements were taken preintervention, and immediately post-intervention. Outcome measures were shoulder flexion, shoulder internal rotation, shoulder external rotation, shoulder horizontal adduction and shoulder abduction using a standardized baseline goniometer instrument. The exclusion criteria for the data analysis were based on the participant's eligibility that was dictated by the following: surgery in either of their shoulders, fractures to either humerus, scapula and or clavicle, history of rotator cuff surgery, history of adhesive capsulitis, labral tear in either shoulder, suffering from unusual sensations, radiating or referred pain, severe shoulder pains month prior to the study, diagnosed with heart disease, type 1 or type 2 diabetes, rheumatoid arthritis or osteoarthritis and or cancer, shoulder 
pain due to neck movements, injections of corticosteroid in the past 3 months, physical therapy within the last 6 months, injury to shoulder or scapula in the previous 3 months that prevented to limit their physical activity to only one day, and any diagnosis of a Long Thoracic Outlet Syndrome, herniation to any cervical/thoracic disc, and or any nerve or connective tissue disorder that could of affect the outcome results. The reported measurements for shoulder range of motion were analyzed using SPSS statistical software (version 22.0; IBM Corporation, Armonk, NY)) providing an independent samples T-test data analysis.

\section{Participants}

Ten male overhead athletes (age $=23.5 \pm 1.5$; height $=$ $186.69 \pm 6.35 \mathrm{~cm}$; mass $=91.86 \pm 12.48 \mathrm{~kg}$ ) were recruited from the Phoenix metropolitan area in Arizona. The average experience in overhead sports such as softball or baseball was $15.5 \pm 4.5$ years (range 11-20 years). Nine of the overhead athletes were right arm dominant, and 1 was left-arm dominant. We defined dominant arm for the subject as which arm they threw a ball.

The inclusion criteria for participant eligibility for participation were active males between the ages of 18-35 years old who participated in a overhead throwing sport for a minimum of 3 months to a year before this study. Before the study, the participants received and completed a Shoulder Health Pre-screening Questionnaire and a Shoulder ROM Questionnaire [7]. The Shoulder Health Pre-Screening Questionnaire is a 19 question self-assessment that allows the participants to be eligible based on the exclusion criteria. The Shoulder ROM Questionnaire is a 3-item self-assessment instrument that instructs the participants to self-examine their ROM (shoulder flexion, external rotation, and internal rotation) before receiving the interventions. Participants that did not meet the criteria were disqualified from this study.

\section{Procedures}

The pre-intervention baseline range of motion collection was conducted in standardized order, which includes shoulder flexion with fixed scapula at $0^{\circ}$, shoulder internal and external rotation at $90^{\circ}$ horizontal abduction and $90^{\circ}$ elbow flexion, horizontal shoulder adduction at $90^{\circ}$ shoulder flexion and shoulder abduction at $90^{\circ}$ shoulder abduction of the participant dominant arm [10]. A single examiner conducted all of the range of motion measurements. All of the range of motion were collected while the patient was in supine. For shoulder flexion, the assistant stabilized the scapula by placing the palm of 1 hand on the anterior-lateral aspect of the scapula. Next using the free arm, the assistant takes the participant's dominant arm through its full passive range of motion until the endpoint was reached. The end motion is defined by Meyers et al., as a cessation of motion or the point at which glenohumeral movement was appreciated [10].

The primary examiner positioned the goniometer and recorded the end-point of the shoulder range of motion. Shoulder range of motion was measured using a standard, long-arm goniometer with a bubble level secured to the stationary arm to assist with device alignment [10]. Anatomical landmarks for goniometer alignment and the patient position for measurements was referenced by the fourth edition text of the Examination of Musculoskeletal Injuries [10]. Need to re-write this sentence, unclear: Each participant was positioned supine on the edge of the ability of their dominant throwing limb where the participants' scapula was in a fixed position [10]. All goniometer measurements were recorded in the same order for all participants to prevent inconsistency of measurement protocol. Shoulder flexion was measured with the axis at the central head of the humerus with the stationary arm at the midline of the trunk and the movement arm along the long axis of the humerus. The limb was then moved overhead in the upward direction to end range [10]. Measurements of internal and external rotation followed with the patient's shoulder placed in 90 degrees of horizontal abduction with the elbow at 90 degrees of flexion [10]. Internal rotation was measured first followed by external rotation with the axis of the goniometer at the olecranon process of the elbow, and the stationary arm positioned perpendicular to the table and the movement arm at the long axis of the ulna [10]. The horizontal abduction was recorded followed horizontal abduction to complete the range of motion measurements. The axis of the goniometer was placed at the superior aspect of the acromion process, with the stationary 
arm perpendicular to the table and the movement arm placed on the long axis of the humerus [10].

The participants were randomly assigned either to the Swedish massage $(n=5)$ or the traditional passive stretching protocol group $(n=5)$. The duration of each intervention was a standardized time of five minutes. The intervention was immediately administered after the pre-intervention range of motion data collection. The Swedish massage group received a 5-minute duration of Swedish therapeutic massage, consisting of the effleurage technique, with coconut butter lotion, and for participants allergic to coconut, a non-perfumed lotion [11]. Swedish massage focused on deep tissue myofascial release, positional release, passive and resisted joints mobilization and neuromuscular proprioception [11]. The Swedish massage was directed to the teres minor, infraspinatus, and posterior deltoid [11]. Effleurage technique was applied by starting at the insertion of each muscle, in a longitudinal direction along towards the origin of the muscle targeted [11]. There was enough pressure from the examiner's hands to create a slight discomfort for the participant [11].

Participants in the traditional passive stretching protocol group $(n=5)$ were placed in the supine position with their dominant throwing limb at the edge of the table. The participants were then placed through five distinct passive stretching positions. The five stretching positions were modified from the twelve shoulder stretching positions examined in Fauls Stretching Routine conducted in a previous study testing the acute effects of shoulder range of motion in the colligate baseball athletes after a twelve phase stretching protocol [12]. The original protocol was condensed into five stretching phases and increased from 10 second passive static holds to 30 -second passive static holds. The passive static stretching routine was manually performed by the primary examiner placing the participants' limb in the flexed, externally rotated, internally rotated, horizontal adduction and abduction positions twice just past end range to the point of participant discomfort and held for 30 seconds for a completed standardized time of five minutes [12].

\section{Statistical Analysis}

SPSS version 17 (SPSS, Inc., Chicago, IL) was used for statistical analysis. For the measurements recorded comparing the differences in shoulder range of motion postintervention vs. pre-intervention of Swedish massage and a passive static stretching routine, we used Independent Samples T-test. Independent Samples T-test was applied due to the studies association of variables13, i.e., Swedish massage and passive static stretching group and the specific use of a single population in overhead throwing athletes [13]. The assumptions that allowed this study to use Independent Samples T-test were the continuous variable for both pre- \& post-interventions are independent, the participants were randomly sampled, and each intervention had a minimum of 5 participants, and there were no outliers. Levene's test for equality of variances was applied due to equal sample sizes in both intervention groups, allowing the assumption of homogeneity of variances [13]. The homogeneity of variance provides an F-statistic and a significance value ( $\mathrm{p}$-value). We used the $p$-value $(p>0.05)$ to determined that both groups can be seen as equal. However, if the groups are unequal ( $p$ $<0.05$ ), then the variances have violated the assumption of homogeneity of variances allowing us to determined which therapeutic intervention had a statically significance [13]. Based on our results of Levene's Test for Equality of Variance, we can reject the null hypothesis, which is that there is no difference between participates that received Swedish massage or a traditional passive stretching protocol in shoulder flexion, abduction, adduction, internal rotation and external rotation. We used Sig. (2-tailed) to determine to see which group means in the respected shoulder range of motion are statistically significant. To determine which therapeutic modality was substantial in a shoulder ROM, the Sig (2-tailed) value must be less than 0.05 . To establish the experiments validity, interventions and recorded values were administered by the same clinician for all participants.

\section{Results}

To test the efficacy of which therapeutic intervention Swedish massage and a Traditional passive stretching protocol in overhead throwing athlete's ROM in shoulder flexion, abduction, adduction, internal rotation and external 
rotation, an independent sample t-test was conducted. This study found that Swedish Massage was statistically significant in increasing shoulder internal rotation and increasing shoulder external rotation in Table 1. This study also found that traditional passive stretching protocol was statically significant in increasing shoulder internal rotation and increasing shoulder external rotation in Table 1 . There was no statistical significance in shoulder flexion $(\mathrm{p}=.898)$, abduction $(\mathrm{p}=.621)$, and adduction $(\mathrm{p}=.367)$. These results indicate that participants in the Swedish massage therapeutic intervention group had significantly greater improvements in their internal rotation range of motion post-treatment than the traditional passive stretching protocol group. The study also indicates that participants in the traditional passive stretching protocol had a significantly greater increase in shoulder external rotation than the Swedish massage group.

Table 1. Mean ( \pm standard deviation) of Change in Shoulder ROM between Intervention

\begin{tabular}{lcccc}
\hline & \multicolumn{2}{c}{ Intervention $\left(^{\circ}\right)$} & & \\
\cline { 2 - 3 } ROM & SM & TPS & $p$ & Effect Size $(95 \%$ CI) \\
\hline Flexion & $4.2 \pm 1.6$ & $4.0 \pm 1.6$ & 0.84 & $0.13(-2.53,2.13)$ \\
Abduction & $4.4 \pm 1.4$ & $4.6 \pm 0.9$ & 0.78 & $0.17(-1.51,1.91)$ \\
Adduction & $7.4 \pm 1.8$ & $8.2 \pm 1.5$ & 0.46 & $0.50(-1.62,3.21)$ \\
Internal & $5.2 \pm 1.3$ & $2.2 \pm 0.5$ & 0.001 & $3.05(-4.43,-1.56)$ \\
Rotation* & & & & \\
External & $2.0 \pm 1.0$ & $11.2 \pm 2.7$ & 0.0001 & $4.52(6.23,12.12)$ \\
Rotation* & & & & \\
\hline
\end{tabular}

ROM = Range of Motion; SM = Swedish Massage; Traditional Passive Stretching

*Significantly different $(p<0.05)$

\section{Discussion}

Overhead athletes who participate in overhead sports activities such as softball, baseball, tennis, racquetball, and volleyball are exposed to repetitive loads and strains throughout the overhead motion [1]. Imposed loads on the shoulder can cause symptoms of pain, discomfort, and delayed muscle soreness directly contributing to decreases in shoulder range of motion [3,7] Losses in shoulder range of motion have been previously correlated to diminished athletic performance and a higher probability of suffering from acute or chronic injuries [3]. Opposed to the professional or collegiate athletic populations, overhead athletes are limited in the therapeutic interventions at their disposal [1]. Therefore, overhead athletes should be introduced to more appropriate therapeutic interventions proven to prevent losses in shoulder range of motion.

The use of static stretching is widely used amongst the active population as a preventative strategy to injury through increases in muscle extensibility assisting in improvements in the joint range of motion [8,9] Previous research has shown static stretching to be most effective when the stretch is held between 15 and 30 seconds for a total of two and four repetitions [14] Further, although it is most useful within these parameters, static stretching elicits mild discomfort which can discourage individuals from completing the stretching routines $[9,14]$

Swedish massage is commonly utilized for its sedative effects in reducing muscle tension and pain [11]. The technique has been proven to increase the circulation to the muscles being passively activated contributing to decreases scar tissue adherence and development, myofascial compression, and muscle spasm $[11,15]$. Swedish massage is believed to increase joint range of motion through the decreasing of neuromuscular excitability by desensitizing of Golgi-tendon muscle fibers [15]. The theory behind the impact on muscle tension and how it correlates to an increased range of motion has yet to be confirmed.

This studies aim was to distinguish whether the therapeutic intervention of Swedish massage has equal or more significant effects in shoulder range of motion in the overhead athlete. Results of the study demonstrated Swedish massage group has a statistically significant increase in the acute glenohumeral internal rotation while the passive static stretching was statistically significant with an increase in external rotation. The remaining shoulder motions, horizontal abduction, adduction, and flexions showed no statistical significance for either intervention.

Swedish massage showed a significant increase in internal shoulder rotation compared to the passive static stretch group. Application of Swedish massage was only performed to the posterior portion of the shoulder complex. The lack of internal 
shoulder rotation has been linked to increases in posterior shoulder tightness in the overhead athlete population [16]. Posterior capsule tightness is thought to be due to fibrotic contracture or the shortening of muscles or scar tissue most commonly seen in a population of repetitive microtrauma [17]. The increase scar tissue and collagen alignment prevent the head of the humerus from translating through its axis or rotation mostly inhibiting internal rotation [17]. Swedish massage has been used clinically to decrease scar tissue formation through its increase in blood circulation $[11,15]$. The application of Swedish massage exclusively to the posterior capsule of the shoulder may have contributed to the statistical significance. The study additionally substantiated a statistically significant increase in only external rotation for the passive static stretching group. The increase in external rotation within the static stretch group compared to the Swedish massage group may be contributed to the lack of shoulder surface treated. External rotation of the shoulder will cause pressure to the anterior chamber of the glenohumeral joint which was not focused on in the Swedish massage group.

We acknowledge there are limitations within the design of the study. The sample size of the 10 participants is small, which can contribute to significant and insignificant findings within the study. Second, this study only focused on male participants between the ages of 22 and 25 who were involved in overhead softball. Further investigators comparing Swedish massage to static stretching routines should test multiple overhead sports populations and diversify the participant pool. Lastly, application of Swedish massage should be longer and expanded to all portions of the shoulder complex. This study focused on the posterior capsule of the shoulder and neglected the anterior, potentially contributing to the lack of significance in the horizontal abduction, adduction, and flexion shoulder motions.

\section{Conclusion}

In conclusion, this study provides an introduction to the potential acute effects of Swedish massage on shoulder range of motion in overhead athletes. Swedish massage had equal to or greater improvement results in four out of the five shoulder motions measured within the study. Swedish massage provided statistically significant change in the participant's shoulder internal rotation when compared to the passive static stretching group which may be correlated to decreased posterior shoulder tightness. Static stretching still proved to have a greater effect on the shoulder external rotation. Overhead athletes should be educated on the potential beneficial effects of massage therapy and its role in maintaining shoulder range of motion.

\section{Conflicts of Interest}

The authors declare no conflict of interest. All authors indicate that no benefits in any form have been received or will be received from a commercial party related directly or indirectly to the participant of this article.

\section{References}

1. Lo YP, Hsu YC, Chan KM. Epidemiology of shoulder impingement in upper arm sports events. Br J Sports Med. 1990; 24(3):173-177.

2. Shanley E, Michener LA, Ellenbecker TS, Rauh MJ. Shoulder range of motion, pitch count, and injuries among interscholastic female softball pitchers: a descriptive study. Int J Sports Phys Ther. 2012; 7(5):548-557.

3. Nelson N. Delayed onset muscle soreness: is massage effective?. J Body Mov Ther. 2013; 17(4):475-82.

4. Bailey LB, Thigpen CA, Hawkins RJ, Beattie PF, Shanley E. Effectiveness of manual therapy and stretching for baseball Players with shoulder range of motion deficits. Sports Health. 2017; 9(3):230-237.

5 . Brummitt J. The role of massage in sports performance and rehabilitation: current evidence and future direction. $\mathrm{N}$ Am J Sports Phys Ther. 2008; 3(1):7-21. PubMed.

6. Jun JY: The effects of kyongrak massage in the elderly with chronic pain. Korean J Rehabil Nurs. 2001; 4:155-164.

7. Yeun Y-R. Effectiveness of massage therapy on the range of motion of the shoulder: a systematic review and metaanalysis. J Phys Ther Scie. 2017; 29(2):365-369.

8. KENDALL BJ. The acute effects of static stretching 
compared to dynamic stretching with and without an active warm up on anaerobic performance. Int. J Exercise Scie. 2017; 10(1):53-61.

9. Samson M, Button DC, Chaouachi A, Behm DG. Effects of dynamic and static stretching within general and activity specific warm-up protocols. J Sports Scie \& Med. 2012;11(2):279-285.

10. Shultz S, Houglum P, Perrin D. Examination of Musculoskeletal Injuries. 4th ed. Grensboro, NC: Human Kinetics.

11. Paul A. van den Dolder, Paulo H. Ferreira, Kathryn M. Refshauge; Effectiveness of Soft Tissue Massage for Nonspecific Shoulder Pain: Randomized Controlled Trial. Phys Thera. 2015; 95(11):1467-1477.

12. Sauers E, August A, Snyder A. Fauls stretching routine produces acute gains in throwing shoulder mobility in collegiate baseball players. J Sport Rehabil. 2007; 16(1):28-40.
13. Armstrong LE, Kraemer WJ. ACSM's Research Methods. Philadelphia, PA: American College of Sport Medicine. 2016.

14. Page P. Current concepts in muscle stretching for exercise and rehabilitation. Intl J Sports Phys Ther. 2012; 7(1):109-119.

15. Mustafa K, Furmanek MP, Knapik A, Bacik B, Juras G. The impact of the Swedish massage on the kinesthetic differentiation in healthy individuals. Int J Thera Mass \& Body. 2015; 8(1):2-11.

16. Laudner KG, Sipes RC, Wilson JT. The Acute Effects of Sleeper Stretches on Shoulder Range of Motion. J Athl Train. 2008; 43(4):359-363.

17. Dashottar A, Borstad J. Posterior glenohumeral joint capsule contracture. Shoulder \& elbow. 2012; 4(4) 4:155-164. 